\title{
A Low-SWaP, Low-Cost Transceiver for Physically Secure UAV Communication with Visible Light
}

\author{
Burak Soner, Sinem Coleri Ergen \\ Electrical and Electronics Engineering, Koç University, Istanbul, Turkey \\ \{bsoner16, sergen\}@ku.edu.tr
}

\begin{abstract}
Unmanned aerial vehicles (UAV) are expected to utilize optical wireless technologies alongside radio frequency technologies for reliable, secure and high bandwidth communications. While terrestrial and atmospheric laser-based solutions in the past have achieved physically secure communication with very complex beam tracking/pointing mechanisms with large and costly telescopes, such systems are neither suitable nor necessary for medium-range $(<100 \mathrm{~m})$ commercial UAV communications. With the proliferation of low-cost solid-state lighting equipment and visible band photodetectors, visible light communications (VLC) offer a low-sizeweight-and-power ( $\mathrm{SWaP}$ ) and low-cost solution. This paper presents a novel lowSWaP and low-cost transceiver for physically secure VLC in medium-range commercial UAV applications. Full implementation details for a proof-of-concept prototype built completely with off-the-shelf components are also reported.
\end{abstract}

\section{Introduction}

Unmanned aerial vehicles (UAV) require reliable, secure and high bandwidth wireless communication with other UAVs and ground stations, both for fulfilling a variety of mission requirements and for maintaining safe, collision-free flight [1]. In order to better fulfill these requirements for a wider variety of environmental and channel conditions, radio frequency (RF) communications for UAVs can be complemented by optical wireless communications (OWC) [2]. OWC refers to use of the optical band ( 200-1600 nm) for communication purposes. Compared to its RF counterpart, OWC offers higher and unregulated bandwidth and higher robustness to electromagnetic interference. Predominantly a line-of-sight (LoS) technology, OWC additionally achieves highly directional, physically secure beams at much lower size-weight-and-power (SWaP) and cost figures compared to RF. A detailed introduction to OWC is provided in [3].

Physically secure communication systems require most or all the energy emanating from the transmitter (TX) to reach the intended receiver's (RX) antenna through a LoS path, leaving practically no energy for non-LoS eavesdroppers. Although this provides the ultimate security, these systems require very precise $(<\mu \mathrm{rad}$ 
[4]) acquisition, tracking and pointing (ATP) in order to keep the TX and RX within each other's LoS, especially for mobile TX and RX. Prior art has thoroughly investigated the ATP problem for a subset of OWC which deals with very long-range terrestrial, aerial, satellite and lunar communications using visible $(\sim 390-750 \mathrm{~nm})$ or near IR band ( $\sim 750-1600 \mathrm{~nm})$ lasers and highly complicated low-divergence optics: free-space optical communications (FSO) [4]. Despite many successful deployments, while still much lower than those of its RF counterpart, the SWaP and cost figures of FSO ATP are still too large for use in medium-range $(<100 \mathrm{~m})$ high volume commercial applications like communications for small-payload UAVs [5]. For such applications, another subset of OWC, visible light communications (VLC), can provide feasible low-SWaP and low-cost solutions.

VLC utilizes the very cheap and highly available visible band light emitting diodes (LED), laser diodes (LD), photodetectors (PD) and optics on the lighting equipment market for versatile design choices in performance, SWaP and cost [6]. Five main challenges have been identified for using VLC in UAV communication in [7]: (i) asymmetry of ground-facing and air-facing links in terms of ambient noise, (ii) high data rate, (iii) long range, (iv) tracking/pointing for high mobility, (v) energy efficiency. While [8] addresses the data rate, range and energy issues for a similar application (inter-satellite VLC) via simulations, the dynamic tracking/pointing issue is not explicitly addressed. The physically secure UAV VLC transceiver proposed in this paper presents a low-SWaP and low-cost solution to the high mobility tracking issue. Furthermore, since the $\%$ of the total TX energy reaching the RX increases with lower beam divergence, the proposed transceiver decreases path loss and increases signal-to-noise ratio and effective range [9], presenting also a solution to the long-range VLC challenge.

This paper initially discusses the system model for medium-range $(<100 \mathrm{~m})$ physically secure UAV communications with VLC in Section 2. Afterwards, it presents the design of a low-SWaP, low-cost VLC transceiver which is feasible for use in this system in Section 3. Full implementation details for a proof-of-concept prototype for this design realized with cheap, off-the-shelf components are provided in Section 4 . While the performance of this prototype does not reflect the full potential of proposed transceiver, it proves that a low-SWaP and low-cost VLC solution to the tracking/pointing problem exists. The paper is concluded in Section 5 after a summary of the presented work.

\section{System Model}

The system model, depicted in Fig.1, comprises of two UAVs, $<100 \mathrm{~m}$ apart, which carry VLC transceivers (TRX) with $360^{\circ}$ rotation pan-tilt-type gimballed pointing mechanisms [10] capable of perfectly synchronous communication with each other as long as energy from the TX beam reaches the RX detector. TRXs contain LEDs with standard radially symmetric polar beam patterns (TX) [11], a photodetector 


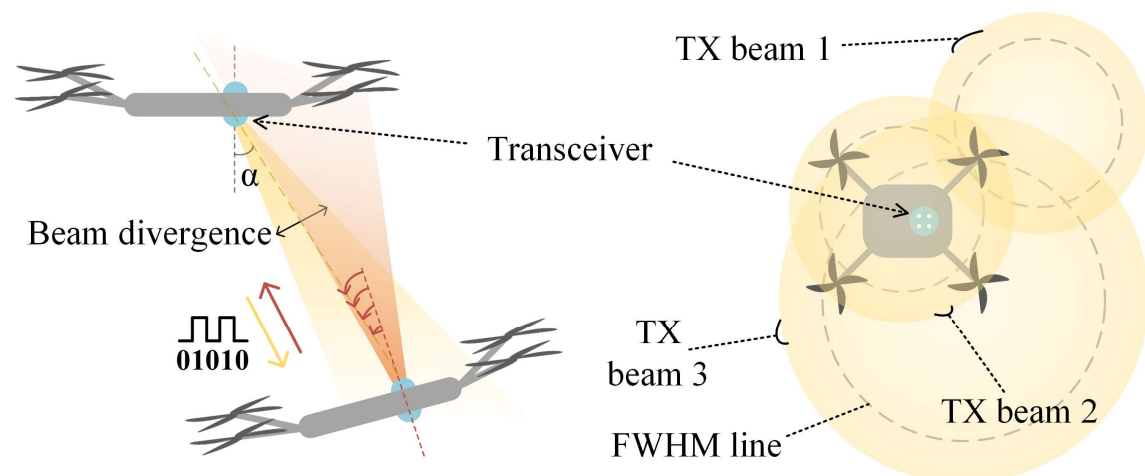

Fig. 1. Investigated system model for medium-range $(<100 \mathrm{~m})$ UAV communications with VLC.

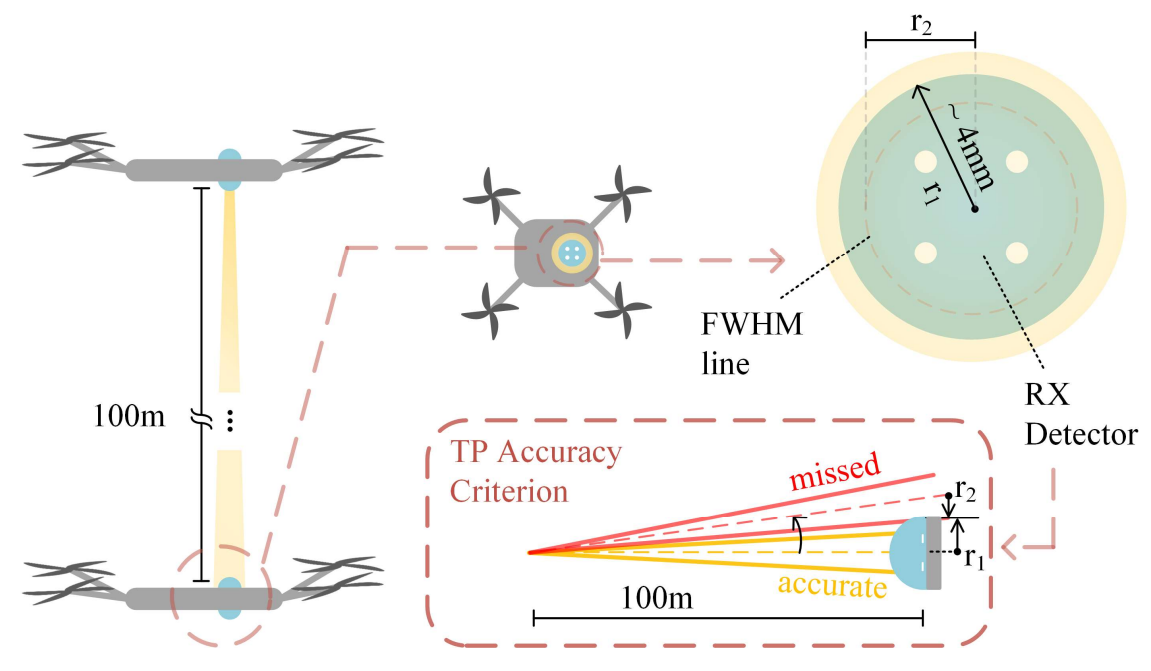

Fig. 2. Tracking/pointing (TP) accuracy requirements for complete physically security at $100 \mathrm{~m}$

$(\mathrm{RX})$, associated drivers and optics and the pan-tilt gimbal mechanism that allows for them to point their LED towards the other's photodetector and track each other's LoS throughout their trajectories for sustained communication.

A tracker/pointer (TP) can be considered to have obtained a LoS link if the RX detector stays within the full width at half maximum (FWHM) of the TX beam footprint on the RX plane, and its accuracy can be measured by how well the beam central axis is aligned with the RX detector surface normal vector. A higher TP accuracy brings higher signal-to-noise-ratio and thus more reliable communication. Furthermore, the physical security level of a beam can be measured by what $\%$ of the total energy emanating from the TX falls onto the RX detector. These concepts are demonstrated in Fig. 1 where TX beam 1 is not in LoS, beam 3 is in LoS but not 
very accurate, and beam 2 has the highest accuracy. Beam 2 is also more secure than 3 since a much larger \% of the total TX beam falls onto the RX detector, leaking out much less energy for potential eavesdroppers. The most stringent TP accuracy requirement for this system occurs when complete physical security (FWHM of TX beam is fully inside RX detector area) is required at the furthest distance $(100 \mathrm{~m})$. This spatial beam confinement requirement, depicted in Fig.2, is common in long-range FSO applications and requires large and costly telescopes and ultrahigh precision TP stages for $>\mathrm{km}$ ranges since the TP accuracy requirement becomes sub- $\mu \mathrm{rad}$. The medium-range UAV VLC application can be treated with much lower SWaP and cost solutions since the required accuracy is inherently lower at smaller distances and furthermore the accuracy requirement can be traded for minor compromises in physical security, like shown in Fig.1.

It is not uncommon for the two TRX in FSO and VLC links to lose LoS due to bad TP or environmental conditions. When LoS is lost between two TRX, an acquisition mechanism is necessary to regain LoS and resume TP for sustained communication. The requirements for acquisition mechanisms vary too much depending on the individual application requirements and are thus left out of this investigation. A summary on state-of-the-art acquisition methods can be found in [4].

\section{Proposed Low-SWaP, Low-Cost Transceiver}

This Section proposes a transceiver which can be used for physically secure VLC between UAVs in $<100 \mathrm{~m}$ distances described in the previous Section. The lowSWaP and low-cost transceiver utilizes a simple standard VLC TX subsystem and a single photodetector on the RX subsystem for both TP and VLC purposes.

\subsection{Transmitter}

The TX subsystem of the proposed TRX simply consists of the TX LED, its driver circuitry and beam-shaping optics which is either composed of static lenses for a given beam divergence, or a set of moveable lenses in order to accommodate for different conditions with dynamic beam divergence control. The TX beam contains AC-coupled VLC signals modulated around a DC bias intensity level by methods like Manchester-coded on-off-keying (OOK), frequency shift keying (FSK) or variable-pulse-position (VPPM) to reduce message-content-dependent flicker [6].

\subsection{Receiver}

The RX subsystem of the proposed TRX uses a quad photodiode (QPD) which can infer the angle-of-arrival (AoA) of the incoming TX beam from the relative intensity difference on each of its cells. QPDs are widely used for laser beam tracking applications with accuracy requirements on the order of a few $\mu$ rads [12]. Rather 
than sensing the AoA explicitly though, the QPD here is used simply as a differential sensor in the TRX controller which constantly tries to keep AoA at zero by moving the TRX with the pan-tilt and balancing the relative intensity on the QPD cells. To converge the incoming beam onto the QPD as a suitably sized spot [13], a hemispherical lens is used [20], and each QPD cell reading is amplified and fed to the TRX microcontroller for both TP and VLC purposes. The optics for the proposed RX subsystem is depicted in Fig.3. Since the VLC signal content of the TX beam is AC-coupled, the QPD amplifier for each cell is also AC-coupled, rejecting the DC ambient noise and conveying the TX signal for that cell as clearly as possible to the microcontroller (MCU). The MCU then uses the QPD readings for both VLC and TP. This process is explained in detail in Section 3.3.

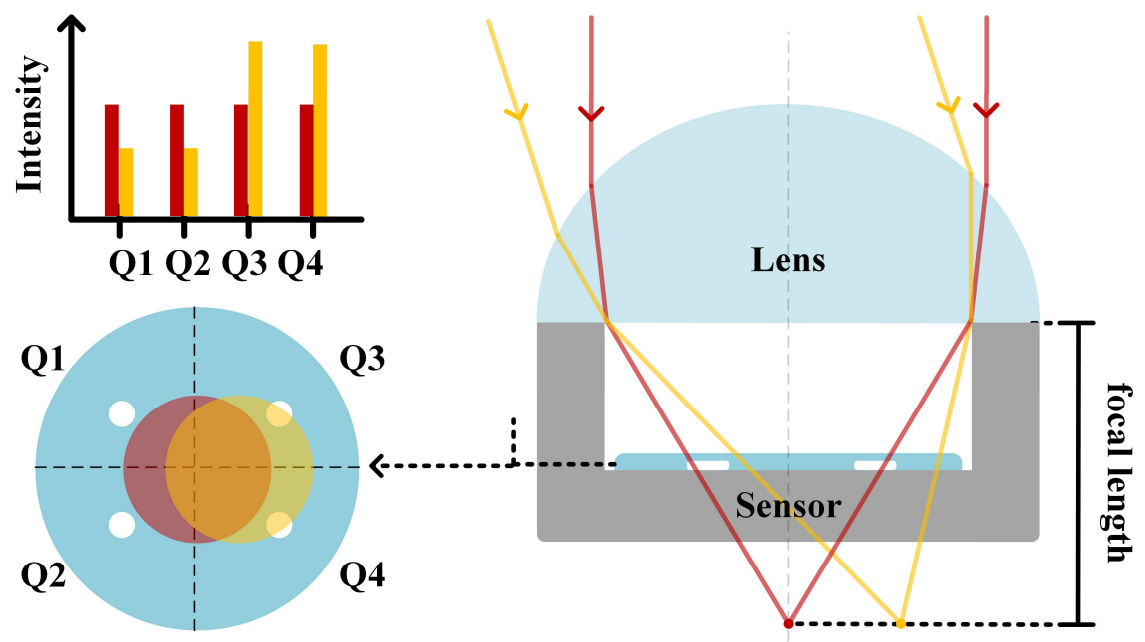

Fig. 3. Receiver optics for the proposed low-SWaP and low-cost transceiver.

\subsection{Tracking and Pointing}

Most long-range FSO ATP solutions separate the communication and ATP subsystems since the two require individual optical designs in order to meet the stringent sub- $\mu$ rad TP requirements. While some previous works have used separate "beacon" beams for TP [14], others have used a single beam for both but separated it with beam splitters and processed the beam for the TP PD and the beam for the communication PD separately [15]. These designs address their application's needs, but they have resulted in high $\mathrm{SWaP}$ gimballed telescopes with high cost. The simple, single-PD-single-beam TRX design proposed in this paper meets the requirements for the application investigated in this paper and is low-SWaP and low-cost.

The QPD in the proposed TRX utilizes the TX beam shining on it for both VLC and TP purposes. Using these QPD cell readings, the microcontroller runs one task for VLC and one task for TP. The VLC task takes the sum of the cells, demodulates 
this to retrieve the VLC message and then responds to the message by modulating the TX LED. The TP task first calculates the signal energy on each QPD cell and deduces the position of the TX beam's spot on the QPD X-y frame. Then it commands the pan-tilt to turn the TRX accordingly to align the TX beam center with the QPD center, achieving accurate LoS tracking between the two TRXs. The functional block diagram for complete TRX operation is shown in Fig.4.

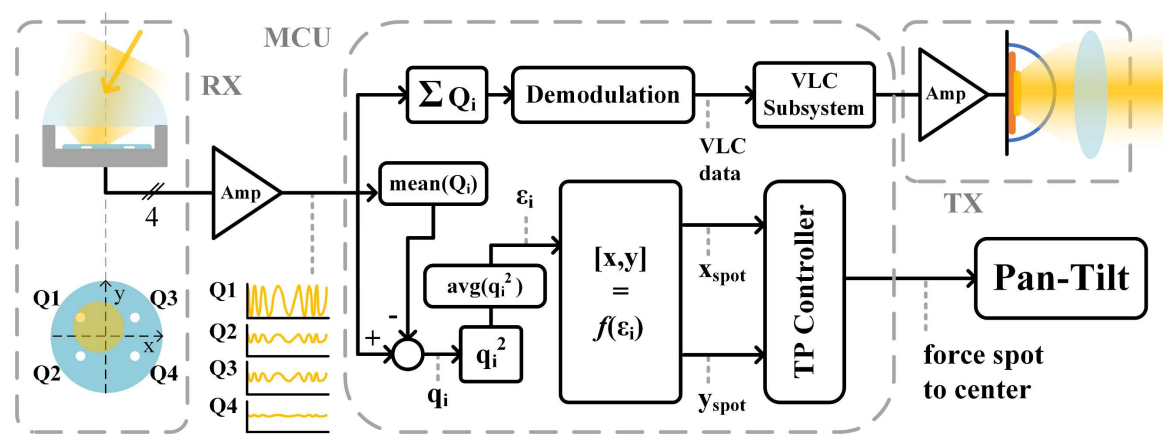

Fig. 4. TRX functional block diagram. Equation (3) is summarized by $[\mathrm{x}, \mathrm{y}]=f\left(\varepsilon_{\mathrm{i}}\right)$. avg: average.

The signal energy for a certain duration $\left(\varepsilon_{i}\right)$ is computed by the TP task by making each $\mathrm{QPD}$ reading for that duration $\left(\mathrm{Q}_{\mathrm{i}}\right)$ zero-mean, taking its square, and calculating the average value of the result:

$$
\varepsilon_{i}=\operatorname{average}\left(\left(Q_{i}-\overline{Q_{i}}\right)^{2}\right)
$$

While increasing the duration over which the signal energy is computed brings better robustness against noise, too large a duration slows the controller down. The signal energies are then used for computing the $\mathrm{x}-\mathrm{y}$ position of the spot on the QPD using the well-known equation for these applications [12], referring to the cell indexing and QPD x-y axis convention shown in Fig.4:

$$
x_{s p o t}=\frac{\left(\varepsilon_{3}+\varepsilon_{4}\right)-\left(\varepsilon_{1}+\varepsilon_{2}\right)}{\varepsilon_{1}+\varepsilon_{2}+\varepsilon_{3}+\varepsilon_{4}} \quad, \quad y_{s p o t}=\frac{\left(\varepsilon_{1}+\varepsilon_{3}\right)-\left(\varepsilon_{2}+\varepsilon_{4}\right)}{\varepsilon_{1}+\varepsilon_{2}+\varepsilon_{3}+\varepsilon_{4}}
$$

For accurate alignment of the TRX LoS, the spot needs to be exactly on the center of the QPD. The TP controller thus always has a zero reference for $\mathrm{x}_{\text {spot }}$ and $\mathrm{y}_{\text {spot }}$ while turning the TRX pan-tilt towards the incoming TX beam center.

\section{Proof-of-Concept Prototype}

This section presents a realization of the transceiver proposed in the previous section using completely off-the-shelf components and provides in-depth implementation details. The prototype is pictured in Fig.5 and a demo video can be found in 
[18]. The prototype consists of one small-scale RX and TX unit built as testing platforms for further designs of complete TRXs. Due to the ultra-cheap components used and visible errors in mounting/build (e.g. Fig.5c, lens/QPD axis skew), performance and TP accuracy of this prototype does not reflect the limits of the proposed transceiver. It's merely an example which demonstrates the single-QPD VLC+TP concept, which is novel for commercial medium-range UAV communications.

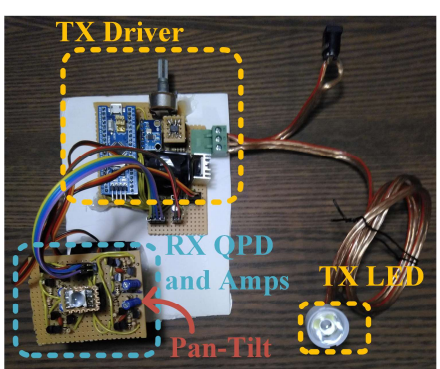

(a)

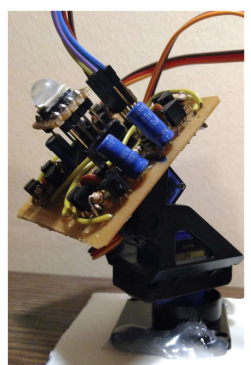

(b)

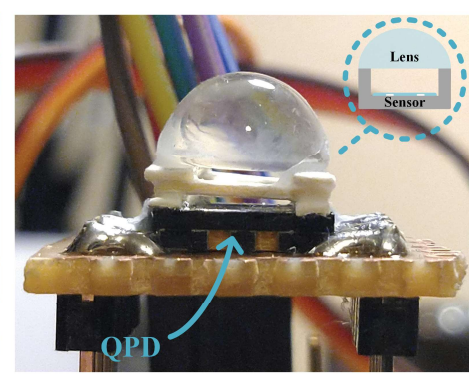

(c)

Fig. 5. Proof-of-concept prototype. TX+RX (a), Pan-tilt (b) and QPD+Lens RX (c).

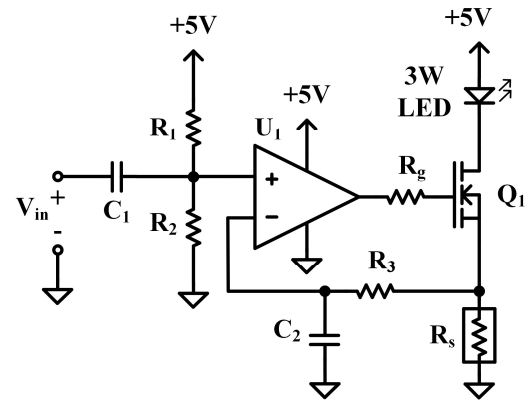

(a)

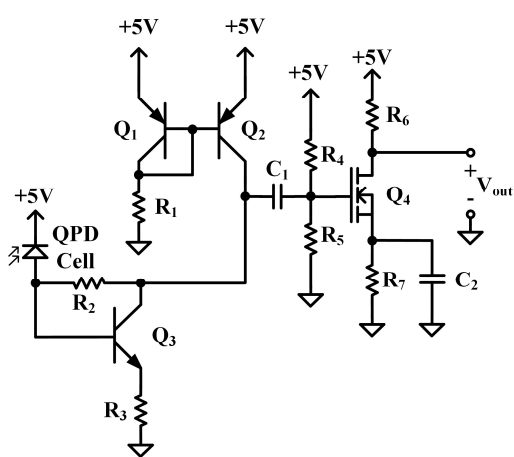

(b)

Fig. 6. Schematics for transmitter (a) and receiver electronics (b).

Table 1. Components for transmitter electronics

\begin{tabular}{|c|c|c|c|c|c|c|c|c|}
\hline $\mathrm{R}_{1}$ & $\mathrm{R}_{2}$ & $\mathrm{R}_{3}$ & $\mathrm{R}_{\mathrm{g}}$ & $\mathrm{R}_{\mathrm{s}}$ & $\mathrm{C}_{1}$ & $\mathrm{C}_{2}$ & $\mathrm{Q}_{1}$ & $\mathrm{U}_{1}$ \\
\hline \multicolumn{2}{|c|}{$100 \mathrm{k} \Omega$ pot } & $100 \Omega$ & $10 \Omega$ & $4.2 \Omega^{*}$ & $10 \mathrm{uF}$ & $100 \mathrm{pF}$ & IRF530 & LMH6643 \\
\hline
\end{tabular}

${ }^{*} \mathrm{R}_{\mathrm{s}}$ is a sandstone resistor

Table 2. Components for receiver electronics

\begin{tabular}{|c|c|c|c|c|c|c|c|c|c|}
\hline $\mathrm{R}_{1}$ & $\mathrm{R}_{2,6}$ & $\mathrm{R}_{3}$ & $\mathrm{R}_{4,5}$ & $\mathrm{R}_{7}$ & $\mathrm{C}_{1}$ & $\mathrm{C}_{2}$ & $\mathrm{Q}_{1,2}$ & $\mathrm{Q}_{3}$ & $\mathrm{Q}_{3}$ \\
\hline $560 \Omega$ & $1 \mathrm{k} \Omega$ & $100 \Omega$ & $100 \mathrm{k} \Omega$ & $200 \Omega$ & $100 \mathrm{nF}$ & $10 \mathrm{uF}$ & $2 \mathrm{~N} 3906$ & $2 \mathrm{~N} 3904$ & $2 \mathrm{~N} 7000$ \\
\hline
\end{tabular}




\subsection{Transmitter}

The transmitter consists of a standard $3 \mathrm{~W}$ white TX power LED [8], a $5^{\circ}$ divergence angle focusing lens [16] and the TX driver electronics. The $5^{\circ} \mathrm{FWHM}$ beam divergence from the source results in a moderate physical security level (beam radius is $8.7 \mathrm{~cm}$ at $1 \mathrm{~m}$ distance). While lower divergence beam-shaping optics are possible with more aggressive off-the-shelf lenses, the lens used in this prototype is a parabolic design with great form factor and holds the LED in place perfectly. The TX design resembles the TX used in [19].

The TX driver schematic and the components used are shown in Fig.5a and Table 1 respectively. The driver is essentially a current servo where input voltage $V_{\text {in }}$ is the reference for the current flowing over the LED. Luminous flux generated by this LED increases linearly with current [11]. The op-amp linearizes the loop dynamics which include the current sense resistor $\mathrm{R}_{\mathrm{s}}$ and the MOSFET. The LED bias intensity, around which the modulation waveform swings, was set manually by a $100 \mathrm{k} \Omega$ potentiometer on $R_{1}$ and $R_{2}$ to fully utilize the LED dynamic range without turn-off or saturation/clipping. $V_{\text {in }}$ was fed from an AD9833 Digital Direct Synthesizer (DDS) which generated the binary FSK (BFSK) waveform that modulated the LED intensity for encoding VLC messages. The BFSK symbols used were $3 \mathrm{kHz}-$ $>0$ and $2 \mathrm{kHz}->1$ with a bit-rate of $1 \mathrm{kbps}$, and the output voltage swing was 0.7

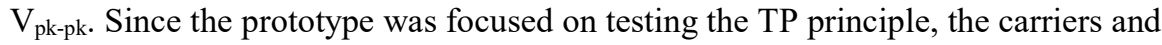
bit-rate were kept moderately low for ease on the MCU side and random data was transmitted. With higher-end MCUs, higher bandwidth communication is possible.

\subsection{Receiver}

The receiver consists of a QPD with SMD package, OPR5911 from TT Electronics, a hemispherical lens of diameter $8 \mathrm{~mm}$ and focal length $5 \mathrm{~mm}$ mounted $2 \mathrm{~mm}$ above it and a cascade AC-coupled transimpedance and voltage amplifier stage. The RX schematic and the components used are shown in Fig.5b and Table 2 respectively.

\subsection{Tracking and Pointing}

The Adafruit mini pan-tilt kit with standard "micro-servos" [17] (angular resolution of $0.5^{\circ}$ ) was chosen for the TP subsystem since the prototype was simply built for testing the principles set forth in the previous Sections using the QPD. An STM32F103C8T6, commonly known as the "blue pill", was used as the MCU. The MCU has a 12-bit ADC which was clocked at 50kSPS. Following the procedure described in Section 3.3 and Fig.4, the MCU takes a 400-sample buffer from each QPD (corresponding to $8 \mathrm{~ms}$ ), subtracts the mean, takes the square, finds the average of the buffer for each QPD for that $8 \mathrm{~ms}$ duration, which is then used for finding $\mathrm{X}_{\text {spot }} / \mathrm{y}_{\text {spot }}$. A simple proportional controller then tries to keep the spot on the QPD center. While $<8 \mathrm{~ms}$ buffers gave faster TP response, values that approached the full 
wave periods of BFSK carriers created oscillating average values, hence oscillating $\mathrm{X}_{\text {spot }} / \mathrm{y}_{\text {spot }}$. Larger buffer sizes gave better stability and noise resilience but the MCU memory limited the size to $15 \mathrm{~ms}$. Pan axis tracking performance results are shown in Fig. $7 \mathrm{a}$ and Fig.7b, demonstrating this effect. Since the tilt axis performs in a similar manner (i.e. only gravity effects differ), only the pan axis results were shown. Performance for different buffer sizes under stationary and dynamic beam TP conditions are shown explicitly in the demo video [18].

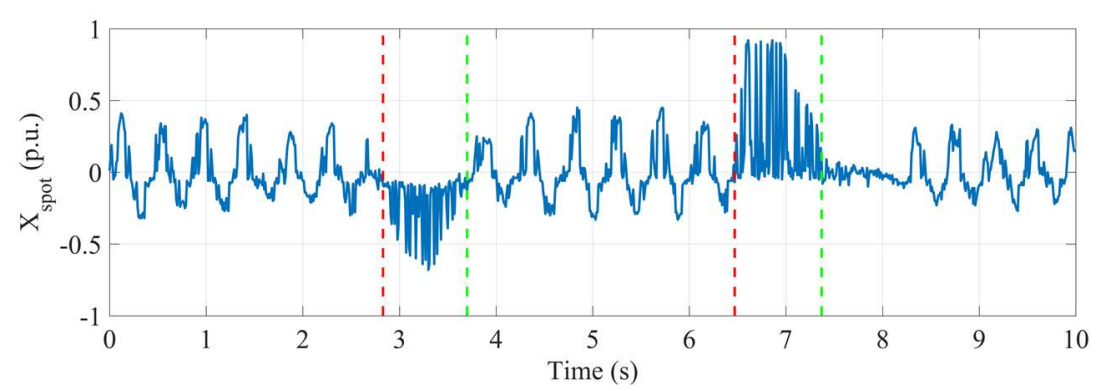

(a)

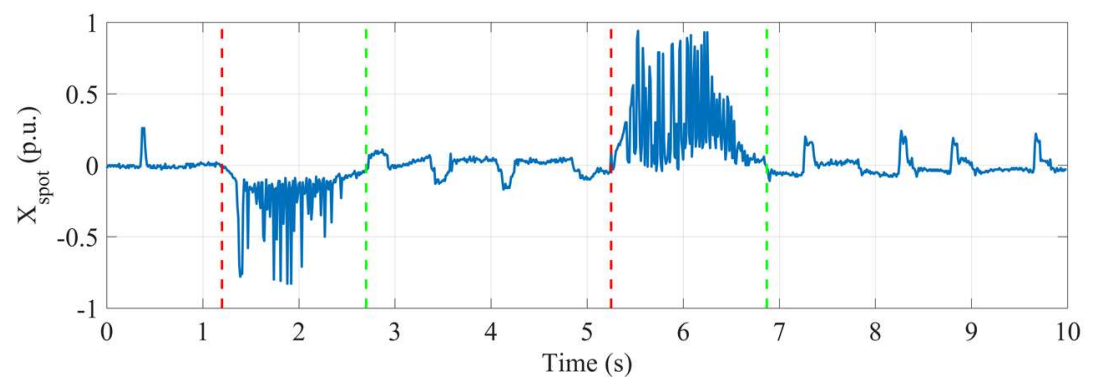

(b)

Fig. 7. Pan axis control performance with buffer sizes of $2 \mathrm{~ms}(\mathrm{a})$ and $10 \mathrm{~ms}(\mathrm{~b})$ demonstrated via the QPD spot x-position in time. Red lines are where step changes in TX beam direction occur and green lines are where the controller settles. Same step change was applied in both cases. While settling time is $\sim 0.9 \mathrm{~s}$ in (a) and $\sim 1.8 \mathrm{~s}$ in (b), the steady state behaviour of (a) is very oscillatory since the buffer size is close to the full wave period of the TX VLC carrier waveform.

\section{Conclusion}

This paper presented a novel low-SWaP and low-cost transceiver (TRX) for physically secure visible light communication (VLC) in medium-range commercial UAV applications. To realize the design in low-SWaP, a single quad photodiode was used on the RX end both for VLC and tracking/pointing (TP) purposes. A proof-of-concept prototype using only off-the-shelf components was realized and demonstrated. While the basic prototype does not reflect the performance limits of the proposed TRX, it conveys the basic principle of the single-beam-single-detector VLC+TP. 


\section{Acknowledgement}

The authors would like to thank Kıvanç Hedili from Koç University, Istanbul, Turkey for his contributions on the design of the RX subsystem.

\section{References}

[1] Y. Zeng, R. Zhang and T. J. Lim, "Wireless communications with unmanned aerial vehicles: opportunities and challenges," in IEEE Communications Magazine, vol. 54, no. 5, pp. 36-42, 2016.

[2] C. Chlestil et al, "Reliable Optical Wireless Links within UAV Swarms," 2006 International Conference on Transparent Optical Networks, Nottingham, 2006, pp. 39-42.

[3] M. Uysal and H. Nouri, "Optical wireless communications - An emerging technology," 2014 16th International Conference on Transparent Optical Networks (ICTON), Graz, 2014, pp. 1-7.

[4] Y. Kaymak et al, "A Survey on Acquisition, Tracking, and Pointing Mechanisms for Mobile FreeSpace Optical Communications," in IEEE Communications Surveys \& Tutorials, vol. 20, no. 2, pp. 1104-1123, Secondquarter 2018

[5] H. Haan, M. Gerken and M. Tausendfreund, "Long-range laser communication terminals: Technically interesting, commercially incalculable," 2012 8th International Symposium on Communication Systems, Networks \& Digital Signal Processing (CSNDSP), Poznan, 2012.

[6] A. Jovicic, J. Li and T. Richardson, "Visible light communication: opportunities, challenges and the path to market," in IEEE Communications Magazine, vol. 51, no. 12, pp. 26-32, 2013.

[7] A. Ashok, "Position: DroneVLC: Visible Light Communication for Aerial Vehicular Networking". in Proceedings of the 4th ACM Workshop on Visible Light Communication Systems (VLCS '17). ACM, New York, NY, USA, 29-30, 2017.

[8] D. N. Amanor, W. W. Edmonson and F. Afghah, "Intersatellite Communication System Based on Visible Light," in IEEE Transactions on Aerospace and Electronic Systems, vol. 54, no. 6, pp. 2888-2899, Dec. 2018.

[9] I. Bekmezci, O. K. Sahingoz, and Ş. Temel, "Flying Ad-Hoc Networks (FANETs): A survey," Ad Hoc Networks, vol. 11, no. 3, pp. 1254-1270, 2013.

[10] M. Khan and M. Yuksel, "Autonomous Alignment of Free-Space-Optical Links Between UAVs", in Proceedings of Hot Wireless '15, Paris, France, pp. 36-40, 2015

[11] Multicomp 3W High Power LED, White. http://www.farnell.com/datasheets/1678766.pdf

[12] R. Carbonneau, J. Dubois, and G. Harris, "An Optical Gun Muzzle Sensor to Improve Firing Accuracy", Proc. SPIE 0661, Optical Testing and Metrology, 1986

[13] L.G. Kazovsky, "Theory of Tracking Accuracy of Laser Systems," Optical Engineering 22(3), 223339, 1 June 1983.

[14] F. G. Walther, S. Michael, R. R. Parenti, and J. A. Taylor "Air-to-ground lasercom system demonstration design overview and results summary", Proc. SPIE 7814, Free-Space Laser Communications X, 78140Y, 24 August 2010.

[15] M. Guelman , A. Kogan, A. Kazarian, A. Livne, M. Orenstein and H. Michalik, "Acquisition and pointing control for inter-satellite laser communications," in IEEE Transactions on Aerospace and Electronic Systems, vol. 40, no. 4, pp. 1239-1248, Oct. 2004.

[16] Lens, PMMA, FWHM beam angle $5^{\circ}$. https://www.direnc.net/5-derece-lens

[17] https://www.adafruit.com/product/1967. Accessed on 04.04.2019

[18] Demo video of the proof-of-concept prototype: https://youtu.be/wQPWQB3W JM

[19] M. Abualhoul, "Visible Light and Radio Communication for Cooperative Autonomous Driving: applied to vehicle convoy", PhD Thesis, MINES Paris Tech, 2016.

[20] J. M. Kahn and J. R. Barry, "Wireless infrared communications," Proceedings of the IEEE, vol. 85, no. 2, pp. 265-298, 1997. 